\title{
Development of Non-Labeled QCM Biosensor for the Detection of $\beta$-Galactosidase: A Comparative Study of Gold and Polystyrene Nanoparticles
}

\author{
Krishna Pal Singh ${ }^{1 *}$, Manav Kumar Choudhary ${ }^{1}$, Iva Chianella ${ }^{2}$, Prashant Singh $^{3}$ \\ ${ }^{1}$ Membrane Biophysics \& Nanobiosensor Research Laboratory, C.B.S H, G. B. Pant University of Agriculture \& Technology, \\ Pantnagar, India \\ ${ }^{2}$ Cranfield Biotechnology Centre, Cranfield Health, Cranfield University, Bedfordshire, England \\ ${ }^{3}$ Department of Chemistry, D.A.V. (PG) College, Dehradun, H.N.B. Garhwal University, Srinagar, India \\ Email: *kps_biophysics@yahoo.co.in, i.chianella.1998@cranfield.ac.uk, prashantraj86@yahoo.com
}

Received February 18, 2013; revised March 19, 2013; accepted March 26, 2013

Copyright (C) 2013 Krishna Pal Singh et al. This is an open access article distributed under the Creative Commons Attribution License, which permits unrestricted use, distribution, and reproduction in any medium, provided the original work is properly cited.

\begin{abstract}
The performance of gold and polystyrene nanoparticles was investigated using quartz crystal microbalance (QCM) as sensor platform; $\beta$-Galactosidase antibody with corresponding antigen was utilized for the immunoreaction. The development of the immunosensor included: 1 ) formation of self assembled monolayers on quartz crystals; 2a) immobilization of $p$-aminothiophenol functionalized gold nanoparticles on carboxyl-terminated self assembled monolayer, or $2 \mathrm{~b}$ ) immobilization of polystyrene nanoparticles on crystals modified with $p$-aminothiophenol self assembled monolayer; 3 ) attachment of monoclonal anti $\beta$-Gal on nanoparticles; and 4) detection of target analyte. The nanoparticles used were synthesized in house and characterized by transmission electron microscopy and infrared spectroscopy. The results revealed that antibodies were strongly attached to functionalized gold nanoparticles; the weaker immobilization of antibodies to polystyrene nanoparticles provoked their detachment during antigen detection. When cross reactivity of polystyrene nanoparticles was checked using a different antigen (Brucella), displacement of antibody was not recorded, demonstrating specificity of the reaction. To the best of our knowledge this is the first direct comparison between behaviors of biosensors developed with two commonly used nanoparticles. The results showed that both nanoparticles produced biosensors capable to detect $\beta$-Gal. Nevertheless biosensors developed using polystyrene nanoparticles are simpler, cheaper and more eco-friendly than those developed using gold nanoparticles.
\end{abstract}

Keywords: QCM Biosensor; $\beta$-Galactosidase; Gold Nanoparticle; Polystyrene Nanoparticle

\section{Introduction}

Enzyme $\beta$-Galactosidase ( $\beta$-Gal) was first sequenced by A. V. Fowler and I. Zabin in 1970 [1]. E. coli $\beta$-Gal is a tetramer of four identical 1023-amino acid chains. Each chain consists of five domains, the third of which is an eight-stranded $\alpha / \beta$ barrel that comprises much of the active site [2]. $\beta$-Galactosidase initiates the breakdown of lactose, the type of sugar found in milk. Lactose requires presence of $\beta$-Gal in order to be reduced to the natural form of sugar, glucose and galactose [3]. Deficiency of intestinal $\beta$-Gal activity causes intolerance to lactose containing food with clinical symptoms as abdominal cramps, diarrhea and bloating. Hence the development of a simple test for detection of $\beta$-Gal is of great clinical importance.

"Corresponding author.
Typically, immunoassays based on enzyme, fluorescent, or radioactive element-labeled secondary antibodies and associated substrates are used for this purpose. Although these techniques are sensitive and accurate, they suffer from drawbacks of complexity in concept and design, time-consuming procedures, use of potential hazards, expensive materials and requirement of skilled personnel. To overcome these limitations, rapid, simplified immunoassay tests have been developed. For example, dot enzyme immunoassays, dot-immunobinding assays, and various particles agglutination tests have been used for the detection of antibodies against a wide range of antigens [4].

An alternative method to monitor antibody-antigens reaction is to use immunosensors. Examples of immunosensors reported in the literature are mainly based on potentiometric, optical, amperometric, and piezoelectric or 
acoustic devices (e.g., quartz crystal microbalance, QCM). These transducers convert immunoreaction events into different physical signals and can sense antigen/antibody concentrations by either indirect competitive or displacement reactions or by direct changes in transducer output. QCM devices are an example of the latter type of system. Antigen-antibody affinity reactions can be identified directly by measuring the frequency change corresponding to mass/density increase/decrease on the gold sensor surface [5-7]. The assay concept and procedure are simple and easy-to-use; any usage of potentially hazardous-labeled materials is eliminated. Biosensors based on piezoelectric transducers, however, are limited by factors such as the need of using purified biomolecules as sensing element, the requirement for the target analyte to have a large molecular weight and the probability to get high level of non-specific binding. Generally, the nonspecific binding caused by the free receptor active residues can be successfully blocked by using either nonactive proteins such as bovine serum albumin (BSA), gelatin or non-charged detergents, milk powder etc., but this does not reduce the non-specific binding caused by the cross interaction between the crude protein and the sample components. Highly purified biomolecules are therefore used to ensure a minimal non specific binding and cross-reactivity. The drawback of the low sensitivity for detection of small molecules could be overcome by modifying the electrodes with nanoparticles. In fact modifications of sensor with nanoparticles increase the surface area, allowing the attachment of a higher amount of recognition element which enhances affinity and sensitivity. Thiol-based chemistry can be used to immobilise nanomaterials (e.g., nanoparticles) on gold surfaces through formation of self assembled monolayers leading to formation of nanofilms. Nanofilms on transducer surfaces have the advantage of allowing easy miniaturization and creation of novel functional interfaces. Selfassembled monolayer of compounds containing thiol groups have shown to be an attractive mean to chemically modify surfaces for various applications like controlling surface wettability, structuring surfaces, binding of species such as metal ions, nanoparticles, and biomolecules [8-11].

QCM biosensors have been developed for the detection of a variety of target analytes such as genetically modified organisms (GMOs) [12], biological warfare agent Francisella tularensis [13]. In addition, a non-labeled QCM biosensor using different nanoparticles has been reported in recent years for the detection of $E s$ cherichia coli and Rhizobacteria $[14,15]$.

The work described here investigates protocols for the modification of QCM gold surfaces starting from self assembled monolayer, followed by immobilization of two different types of nanoparticles and antibody as biological recognition element for diagnostic purpose. Anti
$\beta$-Galactosidase antibodies (anti- $\beta$-Gal) were employed for the detection of the correspondent antigen, $\beta$-Gal. The work describe here also includes methods for the synthesis and characterization of the gold and polystyrene nanoparticles used for the sensor development. The main objective of this work was to investigate whether it is possible to achieve the same QCM sensor behavior with polymeric nanoparticles (in this case polystyrene nanoparticles) as that of metallic nanoparticles (gold nanoparticles). In addition a secondary objective was the development of an immunosensors for $\beta$-Gal. The ability of the resulting immunosensors to detect $\beta$-Gal and its cross reactivity to Brucella antigen were both investigated. Further developments of the biosensors for $\beta$-Gal for the detection of real samples will be carried out in a second stage of the work.

\section{Material and Method}

\subsection{Material Required}

All the glassware used for the preparation of nanoparticles and for experimental work was supplied by Thermo Electron LLS India Pvt. Ltd., Navi Mumbai, India. The chemicals and solvents used in the study were of analytical grade and were supplied by different firms; styrene monomer (Acros, USA), sodium hydroxide (Fisher scientific, USA), sodium dodecyl sulfate (SDS, Himedia, India), amyl alcohol (Himedia, India) potassium persulfate (PP, Fisher scientific, USA), magnesium persulphate (MP, Fisher scientific, USA) and methanol (Himedia, India) were used. Chloroauric acid solution (SRL, India), tetra-n-octylammonium bromide (TOAB; Sigma, USA), sodium borohydride $\left(\mathrm{NaBH}_{4}\right.$, Fisher Scientific, USA), $p$-aminothiophenol (ATP, Sigma, USA) and toluene (Fisher scientific, USA) were used for the synthesis of gold nanoparticles.

Affinity purified monoclonal antibodies (Anti $\beta$-Gal), specific for $\beta$-Galactosidase $(\beta$-Gal) were obtained from Bangalore GeNei, Bangalore, India. N-hydroxylsuccinimide (NHS; SRL, India), 3,3-thiodipropionic acid (Acros, USA), N,N'-Dicyclohexyl carbodimide (DCC; SRL, India), self prepared gold (AuNPs) and polystyrene nanoparticles (PSNPs), Anti $\beta$-Gal and bovine serum albumin (BSA) (Acros, USA) were used for the modification of quartz crystal surface. An aqueous glycerol suspension of $\beta$-Galactosidase ( $\beta$-Gal) from $E$. coli (Sigma-Aldrich, USA) was used as antigen. Methanol and phosphate-buffered saline (PBS), $\mathrm{pH} 7.4$ were used for washing the crystal surface after each step as per requirement. Doubled distilled water (DD water) was used to prepare all the solutions.

\subsubsection{Composition of Different Solution Used}

Piranha solution was used for cleaning the quartz crystals. This is a mixture of concentrated sulphuric acid $\left(\mathrm{H}_{2} \mathrm{SO}_{4}\right)$ and hydrogen peroxide $\left(\mathrm{H}_{2} \mathrm{O}_{2}\right)$ in the ratio of $3: 1(30 \%$ 
v/v). Phosphate buffer saline (PBS) solution, $\mathrm{pH} 7.2$, was used for dilution of antibody and washing purposes. The composition of PBS used was sodium chloride $(\mathrm{NaCl})$ $0.8 \mathrm{~g}$, potassium chloride $(\mathrm{KCl}) 0.02 \mathrm{~g}$, potassium dihydrogen phosphate $\left(\mathrm{KH}_{2} \mathrm{PO}_{4}\right) 0.024 \mathrm{~g}$, disodium hydrogen phosphate $\left(\mathrm{Na}_{2} \mathrm{HPO}_{4}\right) 0.144 \mathrm{~g}$ in $100 \mathrm{ml}$ double distilled water. The $\mathrm{pH}$ of the solution was adjusted with the help of concentrated $\mathrm{HCl}$.

\subsection{Experimental Methods}

\subsubsection{Synthesis of Gold Nanoparticles}

For the synthesis of gold nanoparticles a phase transfer method was followed. Gold nanoparticles functionalized with $p$-aminothiophenol (ATP) were synthesized by reducing a chloroauric acid solution $(1 \mathrm{mM})$ with $\mathrm{NaBH}_{4}$. A scheme of the entire process is reported in Figure 1. In the first step, the gold ions were transferred to an organic phase (toluene) from aqueous phase by stirring in presence of the phase transfer agent TOAB. Transfer of gold ions was confirmed by the yellowish coloration of the organic phase. Then $25 \mathrm{ml}$ of toluene containing ATP was added to the solution of gold ions to form $\mathrm{Au}$ ions@ATP (with free amino groups on their surface). After $1 / 2 \mathrm{hr}$, the temperature was set to $100^{\circ} \mathrm{C}$ with continuous stirring. Under boiling condition, Au ions@ATP reduction was carried out by adding $\mathrm{NaBH}_{4}$. The molar ratio for the $\mathrm{Au}^{+} /(\text {octyl })_{4} \mathrm{NH}_{4}^{+} \mathrm{Br}^{-} /$thiol $/ \mathrm{NaBH}_{4}$ was fixed to $1: 3: 3: 10$. The color changed from pale yellow to wine red (through colorless) indicating formation of gold nanoparticles in the organic phase. The organic phase containing the nanoparticles was separated using a separating funnel.

\subsubsection{Synthesis of Polystyrene Nanoparticles}

The first step in the synthesis of polystyrene nanoparticles (PSNPs) was the removal of the inhibitor from the monomer styrene by shaking thrice with $10 \% \mathrm{NaOH}$ aqueous solution. After separation, the monomer styrene was washed with double distilled water thrice in order to remove traces of $\mathrm{NaOH}$. Then magnesium sulfate $\left(\mathrm{MgSO}_{4}\right)$ was added to the purified styrene in order to remove water. Destabilized styrene was then stored in a freezer at

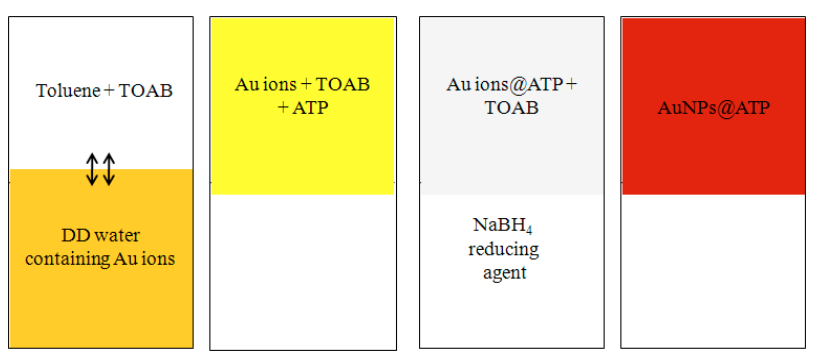

Figure 1. Schematic diagram for synthesis of gold NPs functionalized with $p$-aminothiophenol. $-20^{\circ} \mathrm{C}$.

Microemulsion polymerization technique was used for the synthesis of polystyrene nanoparticles. In order to get a microemulsion, $90 \mathrm{ml}$ of DD water containing $10 \mathrm{mg}$ of SDS and $1.24 \mathrm{ml}$ of amyl alcohol were stirred continuously under nitrogen atmosphere during whole process. $2.5 \mathrm{ml}$ of destabilized styrene monomer was then added with gentle stirring in order to get complete oil dispersion in water (o/w type) emulsion. After that, a potassium persulfate (KPS) initiator solution $(2 \mathrm{ml}$ of $30 \mathrm{mg} / \mathrm{ml}$ prepared in DD water) was added to the reaction mixture maintaining the temperature at $65^{\circ} \mathrm{C} \pm 5^{\circ} \mathrm{C}$. This solution was continuously stirred for $\sim 5 \mathrm{hrs}$ in order to achieve maximum conversion of styrene to PSNPs. The mixture turned white (milky) indicating the formation of PSNPs. In order to precipitate PSNPs, this solution was poured in a large amount of methanol in a ratio of $1: 100$. The PSNPs, precipitated in methanol, were used for further study.

\subsection{Characterization of Nanoparticles (NPs)}

The synthesized nanoparticles were characterized by Transmission Electron Microscopy (JEM 1011, JEOL, Japan) and FTIR (NICOLET 6700, Thermo, USA). Samples for TEM were prepared by evaporating a small drop of particles suspension onto a carbon coated copper grid. For the FTIR data the AuNPs@ATP were diluted in toluene and the colloids of PSNPs were diluted with methanol by $1: 100$. The results were analyzed using origin, the built in software of the FTIR instrument.

\subsection{Biosensor Setup}

The development of biosensor started by cleaning AT-cut quartz crystals, (5 MHz, SRS, USA) with Piranha solution $\left(1: 330 \% \mathrm{H}_{2} \mathrm{O}_{2}: 70 \% \mathrm{H}_{2} \mathrm{SO}_{4}\right)$. The crystals were then rinsed with a large amount of DD water and dried over a stream of $\mathrm{N}_{2}$ gas. These cleaned crystals were then activated chemically through the well known thiolization chemistry $[16,17]$. For this, the crystals were immersed into a methanolic solution of 3,3-thiodipropionic acid (TDPA, $1 \times 10^{-3} \mathrm{mM}$ ) or $p$-aminothiophenol (ATP, $1 \times$ $10^{-3} \mathrm{mM}$ ) in order to get a self assembled monolayer (SAM). The monolayer of TDPA was produced for the immobilization of AuNPs and the ATP monolayer for the immobilization of PSNPs. To get stable and well ordered

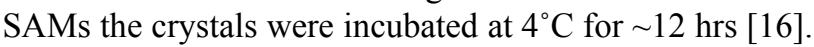
After washing with methanol, the TDPA modified crystal was treated with DCC/NHS $(45 \mathrm{mM} / 15 \mathrm{mM})$ to convert the terminated carboxyl group to an active NHS ester for the immobilization of AuNPs@ATP through their terminal amino group. In the second protocol, the ATP modified crystals were directly incubated with a suspension of PSNPs in methanol at $4^{\circ} \mathrm{C}$ for $\sim 12 \mathrm{hrs}$. 
After rinsing the crystals (in both cases) with methanol and drying, a monoclonal antibody solution, diluted 1:6000 in PBS pH 7.2, was deposited over the crystal surface and incubated at $4^{\circ} \mathrm{C}$ over night $(\sim 15 \mathrm{hrs})$. The excess and/or unattached antibodies were removed from the surface by rinsing with PBS, pH 7.2. The free/untreated and nonspecific sites were blocked by applying $1 \%$ BSA solution in PBS. After rinsing with PBS, the dried crystals were subjected to the antigen, $\beta$-Gal, solution (764 units/mg diluted 1:6000 in PBS, $\mathrm{pH} 7.2$ ) for the detection. The crystal frequency associated with each step in wet as well as in dry state was monitored using a QCM-200 Stanford Research System Inc. USA. For the specificity study of the developed biosensor, after the modification of the crystal as described above, cross reactivity and specificity were investigated using a solution of Brucella antigen ( $5 \mu \mathrm{g} / \mathrm{ml}$ in PBS, $7.2 \mathrm{pH})$.

\section{Results and Discussion}

\subsection{Characterization of Nanoparticles by TEM and FTIR}

The synthesized nanoparticles were characterized with a TEM microscope. Figures 2A and B depicts micrographs of synthesized PSNPs and AuNPs@ATP respectively. The micrograph 2A clearly shows that the nanoparticles are not spherical in shape but have an elliptical shape and an average size of $60 \pm 5 \mathrm{~nm}$ (calculated by histogram, data not shown). It has been reported in literature that polystyrene nanoparticles are spherical when in solution, but might change shape ones dried [18, 19]. Therefore we assume that PSNPs become elliptical after drying on the carbon coated copper grid used for microscopy. Figure 2B is the micrograph of AuNPs functionalized with $p$-aminothiophenol. The particles (the darker spots in the picture) are spherical in shape with a size of $40 \pm 5 \mathrm{~nm}$ (calculated by histogram, data not shown). The grey material in the background is probably due to residual SDS or magnesium persulfate.

Figure 3(a) shows the IR spectra of AuNPs@ATP in toluene. The main peaks in the FTIR spectrum confirm the successful synthesis of the Au particles. The peak at $750 \mathrm{~cm}^{-1}$ represents the substituted benzene ring. The peaks in the range $1450-1500 \mathrm{~cm}^{-1}$ are for aromatic $\mathrm{C}=\mathrm{C}$ bonds. The peak at about $1600 \mathrm{~cm}^{-1}$ shows the presence of the primary amino group. The infrared bands of thiol are usually medium to weak intensity, because of the small dipole moments of S-H bond. The only useful wavenumber for thiol groups is the $\mathrm{S}-\mathrm{H}$ stretching vibration, which is found at $2590-2560 \mathrm{~cm}^{-1}$. The absence of a peak at $\sim 2550 \mathrm{~cm}^{-1}$ confirms the lack of free $\mathrm{S}-\mathrm{H}$ and the peak at about $3025 \mathrm{~cm}^{-1}$ can be perhaps attributed to the thiol-Au bond confirming the attachment of ATP to the nanoparticles.
The chemical structure of the polystyrene nanoparticles prepared was examined by FTIR and results are shown in Figure 3(b). The peak positions obtained at $3025,1493,1369$ and $756 \mathrm{~cm}^{-1}$ were assigned to the stretching vibration of $-\mathrm{C}-\mathrm{H}$ and $\mathrm{C}-\mathrm{C}$ bonds of the phenyl ring, the symmetric stretching of $\mathrm{C}-\mathrm{H}$ bond and rocking vibration of $-\mathrm{C}-\mathrm{H}$ bond in the phenyl ring respectively

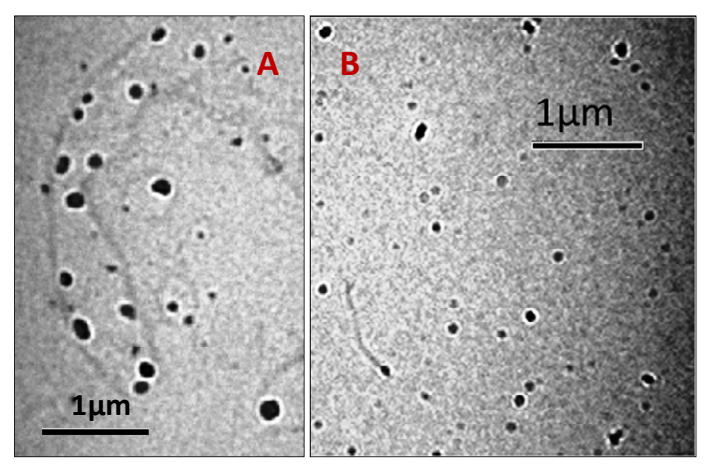

Figure 2. TEM micrograph for the synthesized Nanoparticles (A) for PSNPs and (B) for AuNPs.

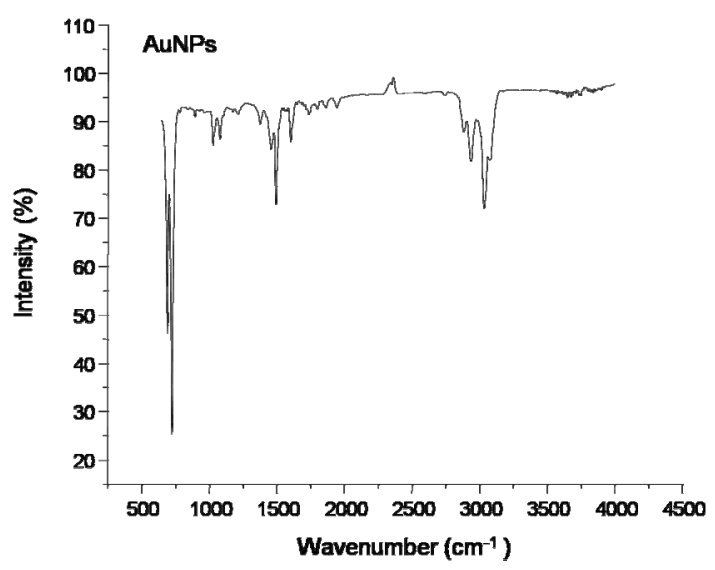

(a)

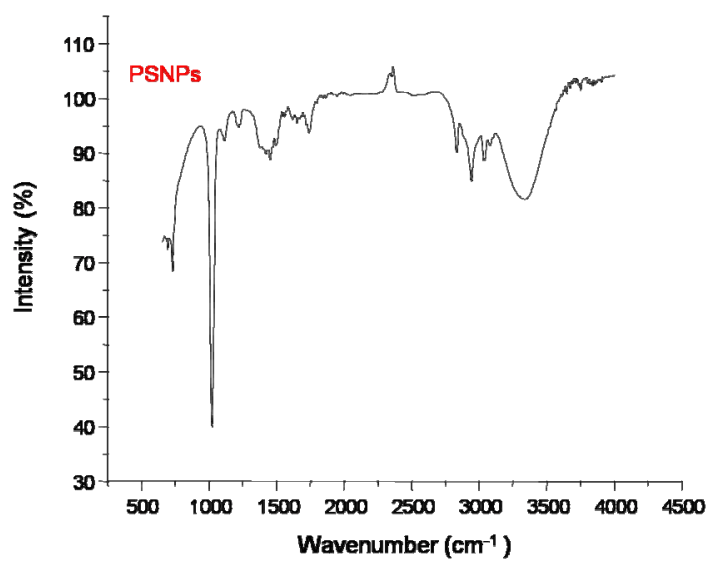

(b)

Figure 3. FTIR spectra for the gold and polystyrene nanoparticles synthesized in laboratory. 
$[20,21]$. The absorption peaks in the range of $700-750$ $\mathrm{cm}^{-1}$, show that the product contains a substituted benzene ring, indicating the presence of styrene. In addition, the absence of absorption peaks for $\mathrm{C}=\mathrm{C}$ in the range $1680-1620 \mathrm{~cm}^{-1}$ confirms the complete polymerization of the monomer styrene [18].

\subsection{Development of Surface Modified Quartz Crystal for Detection of $\boldsymbol{\beta}$-Galactosidase}

The specificity and sensitivity of biosensors depend on many factors. One of these is the method used to modify the sensor surface. In this work two methods were followed for the modification of gold electrodes. The main difference between the two methods was the type of nanoparticles used for the functionalisation: polystyrene nanoparticles (PSNPs) in one case and functionalized gold nanoparticles (AuNP@ATP) in the other. The hypothesis for replacing AuNPs@ATP with PSNPs is that polystyrene should provide a better surface for the immobilization of biomolecules (e.g., antibodies) as it is being already widely used for immunoassay (e.g., in ELISA plates).

\subsubsection{First Protocol: AuNPs@ATP}

The surface modification of AT cut quartz crystals was done in several subsequent steps. The frequency shifts associated with each step was monitored using the QCM system and the results were combined in a single graph. The frequency of quartz crystal, washed with Piranha solution, was firstly recorded in absence of any liquid (in a dry state) and the values considered as the reference frequency for all the dry state monitoring. In absence of liquids the frequency of bare quartz crystal was found almost constant, as shown in Figure 4. An average frequency of $5012209.14 \pm 0.66 \mathrm{~Hz}$ was recorded. A base line for methanol solvent was then obtained by subjecting the crystal to methanol with the use of a batch-equilibrium cell. After the signal stabilization, an average frequency of $5011668.45 \pm 0.81 \mathrm{~Hz}$ was recorded. After this, the surface of the crystals was modified with 3, 3thiodipropionic acid (TDPA). The process was monitored for $\sim 1 \mathrm{hr}$ by recording the frequency using QCM (Figure 4). Then the crystals was disconnected from the QCM and incubated in the TDPA solution for further $\sim 12 \mathrm{hrs}$ at $4^{\circ} \mathrm{C}$. After a wash with methanol, to remove unattached TDPA, the frequency of the dried crystal was monitored and was found to be $5012175.29 \pm 1.25 \mathrm{~Hz}$. This modification allows the attachment of AuNPs@ATP by providing the carboxyl group free and perpendicular to the gold surface.AuNPs@ATP were then immobilized on crystal surface using DCC/NHS chemistry. Treatment with DCC/NHS activates the monolayer by converting the terminal carboxyl groups into active NHS ester groups. These active NHS groups then covalently bind to the primary amine groups of AuNPs@ATP. Once the quartz crystal with the activated carboxylic groups were exposed to AuNPs@ATP solution, the frequency was monitored initially for $\sim 1 \mathrm{~h}$ (Figure 4) and then incubation in the nanoparticles solution was continued for further $\sim 12 \mathrm{hrs}$ at $4^{\circ} \mathrm{C}$ without recording the frequency. After washing and drying the frequency of the crystal was found to be $5012144.33 \pm 0.75 \mathrm{~Hz}$. For the antibody immobilization, the crystal was subjected to a solution of anti $\beta$-Gal antibody prepared in PBS. Monitoring of the resonant frequency in the first hour of antibody immobilization showed a decrement in frequency, Figure 4. The antibody immobilization was then continued at $4^{\circ} \mathrm{C}$ for $\sim 15 \mathrm{hrs}$ without monitoring the frequency. Next, the crystal was washed with PBS in order to remove the unattached antibodies and the frequency of the dried crystal was found to be $5011827.95 \pm 0.20 \mathrm{~Hz}$.

Blocking of free sites was carried out by subjecting the crystal to $1 \%$ BSA for an hour to inactivate free binding site and therefore reduce the non specific binding. Excess of BSA was removed by washing with PBS several times. The average frequency of the dry crystal after blocking was $5011717.41 \pm 0.31 \mathrm{~Hz}$. All the average frequency shifts with the standard deviation for experiments repeated in triplicate are reported in Table 1.

For the antigen detection, $200 \mu \mathrm{l}$ of $\beta$-Gal prepared in PBS (pH 7.2) were applied to the sensor and the binding between antigen and antibody was investigated by monitoring the frequency until the end of the reaction, which was revealed by a signal plateau. It is clear from Figure 4 that initially, in the first $\sim 15 \mathrm{~min}$, there was only a very
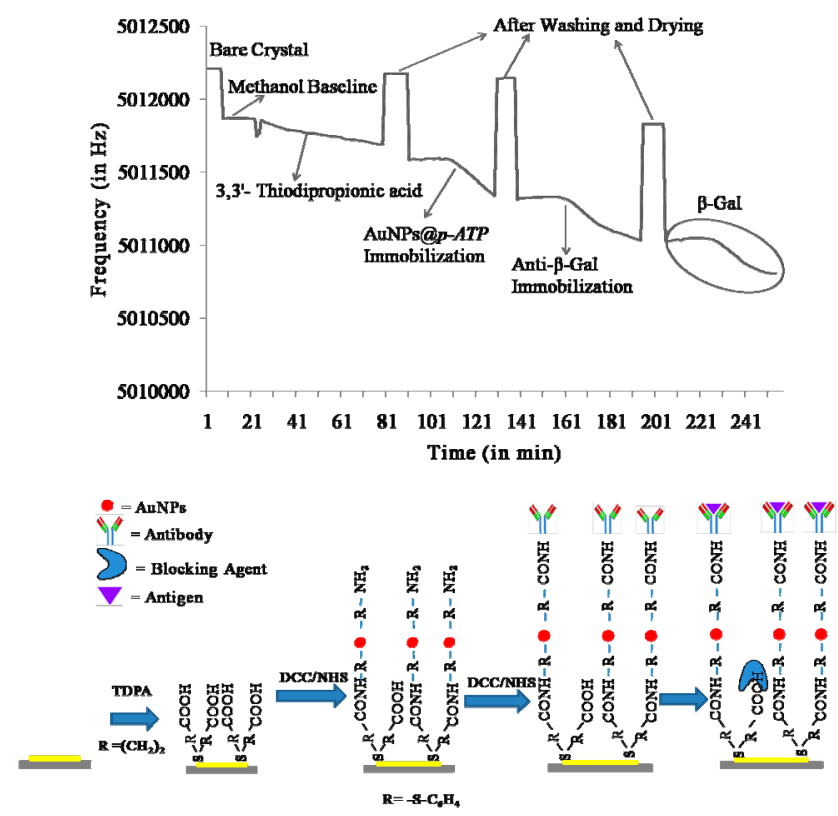

Figure 4. Frequency shift associated with different step when crystal was modified by scheme 1 for the detection of $\beta$-Gal using AuNPs modified crystal. 
Table 1. Average frequency Shift associated with different step during the biosensor development along with standard deviation.

\begin{tabular}{lcccccc}
\hline \multicolumn{1}{c}{ Process } & $\begin{array}{c}\text { AuNPs@ATP } \\
\text { Frequency (Hz) }\end{array}$ & $\begin{array}{c}\text { Average Shift }(\mathrm{Hz}) \\
\pm \text { Std dev }\end{array}$ & $\begin{array}{c}\text { PSNPs } \\
\text { Frequency (Hz) }\end{array}$ & $\begin{array}{c}\text { Average Shift } \\
(\mathrm{Hz}) \pm \text { Std dev }\end{array}$ & $\begin{array}{c}\text { Specificity } \\
\text { Frequency (Hz) }\end{array}$ & $\begin{array}{c}\text { Average Shift } \\
(\mathrm{Hz}) \pm \text { Std dev }\end{array}$ \\
\hline Bare Quartz Crystal & 5012209.14 & $0 \pm 0.66$ & 4995861.85 & $0 \pm 0.69$ & 4995801.09 & $0 \pm 1.53$ \\
Base line Methanol & 5011668.45 & $540.69 \pm 0.81$ & 4995459.86 & $401.99 \pm 1.74$ & 4995394.74 & $406.35 \pm 9.21$ \\
SAM Modification & 5012175.29 & $33.85 \pm 1.25$ & 4995802.24 & $59.61 \pm 0.86$ & 4995757.41 & $43.68 \pm 1.77$ \\
NPs Immobilization & 5012144.33 & $30.96 \pm 0.75$ & 4995736.82 & $65.42 \pm 1.40$ & 4995665.43 & $91.98 \pm 3.50$ \\
Antibody Immobilization & 5011827.95 & $316.38 \pm 0.20$ & 4995233.19 & $503.63 \pm 5.96$ & 4995117.83 & $547.6 \pm 2.78$ \\
Blocking with BSA & 5011717.41 & $110.54 \pm 0.37$ & 4995131.75 & $101.44 \pm 0.42$ & 4994982.03 & $135.8 \pm 3.09$ \\
Antigen Detection & & & 4995725.38 & $-593.63 \pm 0.24$ & & \\
\hline
\end{tabular}

small change in frequency. This might be due to the fact that the antigen requires time to assume the orientation, which exposes a site able to bind the antibody. A sharp decrease was then observed in the next $\sim 20$ min until the sensor reached an equilibrium state shown by a plateau of the signal. The shift in frequency demonstrates the attachment of the antigen $\beta$-Gal to anti- $\beta$-Gal antibody.

The present non-labeled technique has several advantages over traditional immunoassay (e.g., ELISA), in which the results are recorded only at the end of the entire experiment. This is evident in Figure 4 where the interaction between the antigen and the antibody is observed in real time. This information is used for characterization of the immunosensors for $\beta$-Gal.

\subsubsection{Second Protocol: PSNPs}

In this second protocol the surface of the gold electrode was modified with PSNPs instead of AuNPs@ATP. Many of the steps included in this second protocol are the same as in the first protocol. As before the resonant frequency of the several steps was monitored and the results are reported in one graph (Figure 5) and the full protocol is explained below.

The frequency of the bare dry crystal after washing with Piranha solution and methanol was $4995861.85 \pm$ $0.69 \mathrm{~Hz}$. An average frequency of $4995459.86 \pm 1.74 \mathrm{~Hz}$ was obtained after methanol was loaded onto the surface of the crystal (using the QCM cell-holder) and a stable signal was obtained (Figure 5). The surface of the quartz crystal was then modified by subjecting it to a solution of ATP in methanol $\left(1 \times 10^{-3} \mathrm{mM}\right)$. As previously, the process was monitored only initially by recording the frequency for $1 \mathrm{hr}$ using QCM (Figure 5), before a further incubation at $4^{\circ} \mathrm{C}$ for $\sim 12 \mathrm{hrs}$ with the crystal disconnected from QCM. Then the crystal was washed with methanol in order to remove the excess of ATP and the frequency of the dried crystal was found to be $4995802.24 \pm 0.86 \mathrm{~Hz}$. The process was continued with the attachment of PSNPs. The frequency of the crystal exposed to the suspension of PSNPs was again recorded for 1 hour (Figure 5) and the incubation then continued at $4^{\circ} \mathrm{C}$ for other $12 \mathrm{hrs}$ (without monitoring the frequency). After that, the crystal was washed with methanol, to remove the unattached PSNPs, dried and the frequency was found to be $4995736.82 \pm 1.40 \mathrm{~Hz}$, giving a shift of $66 \mathrm{~Hz}$, indicating a successful immobilization of PSNPS. Further decrement in the resonant frequency was observed after immobilization of anti $\beta$-Gal antibody in PBS. As above the resonance frequency was monitored in the first hour of incubation with the antibodies solution (Figure 5) and the immobilization reaction was then continued for $\sim 15 \mathrm{hrs}$. After this, the crystal was washed with PBS (pH 7.2), to remove the excess of antibodies, dried and an average frequency of $4995233.19 \pm 5.96 \mathrm{~Hz}$ was measured. Blocking of remaining active sites of the quartz crystal was carried out with $1 \%$ BSA for an hour. After PBS washing an average frequency of 4995131.75 $\pm 0.42 \mathrm{~Hz}$ was observed.

Thereafter the crystal was subjected to $\beta$-Gal for detection. Previously in the case of AuNPs a decrease in the crystal frequency was observed while in PSNPs case the frequency observations were reverse and surprising. In fact, Figure 5 showed an increase in frequency, which was initially slow but became more pronounced as the time passed. This might indicate removal of mass from

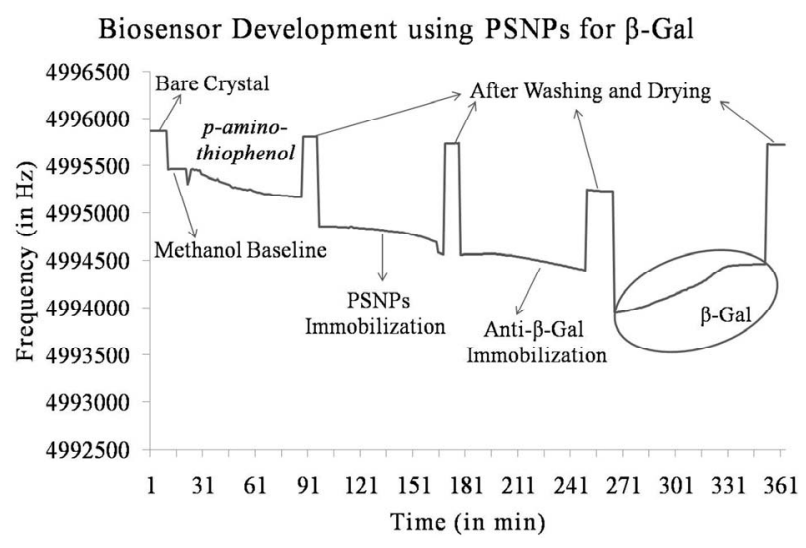

Figure 5. Change in frequency monitored by QCM when biosensor was developed with PS nanoparticles for the detection of $\beta$-Galactosidase. 
the crystal surface. The sensor was kept under investigation for more than an hour. After $\sim 2$ hrs it was disconnected from QCM, washed with PBS, $\mathrm{pH} 7.2$, dried and a frequency value of $4995725.38 \pm 0.24 \mathrm{~Hz}$ was recorded. Total increase in frequency observed after detachment was $593.63 \mathrm{~Hz}$. The frequency $(4995725.38 \mathrm{~Hz})$ is practically equivalent to the average frequency of the crystal after immobilization of PSNPs (4995736.82 Hz), meaning that the antibodies were detached from the sensor surface as they bound the $\beta$-Gal antigen present in solution. All the frequency shifts and corresponding standard deviations observed performing the experiments in triplicate is reported in Table 1.

Probably, since the antibodies are only weakly adsorbed on PSNPs, the presence of the antigen in solution is enough to displace the anti- $\beta$-Gal from the sensor surface $[22,23]$. These polystyrene NPs attached on the crystal surface through physical forces only and not by electrostatic attraction forces. These attraction forces may be imparted by the partially positive $-\mathrm{NH}_{2}$ groups, which has termed as "polarity"; as the polystyrene surface has hydrophobic nature. Consequently, the anti- $\beta$ Gal binds to surface by weak hydrophobic bonds while these molecules tend to bind with water molecules with stronger hydrogen bonds. This was not observed in the case of AuNPs@ATP, as the antibodies were more strongly attached to the sensor surface through amide bonding.

A further control experiment was also performed to confirm the enhancement in efficacy derived by the use of both types of nanoparticles. For this study, the antibody was directly immobilized on ATP SAM modified quartz crystal through DCC/NHS chemistry, without the NPs. In absence of nanoparticles, the frequency shift for the binding of the anti- $\beta$-Gal directly to the ATP monolayer was $130 \mathrm{~Hz}$, instead of the $381 \mathrm{~Hz}$ obtained onto the AuNPs and $629 \mathrm{~Hz}$ obtained onto the PSNPs, proving the enhanced efficacy caused by the nanoparticles.

\subsubsection{Specificity of the Developed Biosensor}

To study the specificity of the biosensor, the sensor surface was prepared using the second protocol (PSNPs) and the anti $\beta$-Gal antibody. The several steps involved in the biosensor preparation are summarized below: Firstly, the resonant frequency of the crystal after washing with Piranha and methanol was $4995801.09 \pm 1.53$ $\mathrm{Hz}$ (Figure 6). An average frequency value of $4995394.74 \pm 9.21 \mathrm{~Hz}$ was obtained after stabilization of the crystal signal when exposed to methanol. After this, the surface of the quartz crystal was activated with $1 \mathrm{mM}$ ATP solution in methanol. The process was monitored using the QCM system for $\sim 1 \mathrm{hr}$ (Figure 6) and then continued at $4^{\circ} \mathrm{C}$ for $\sim 12 \mathrm{hrs}$, without monitoring. The

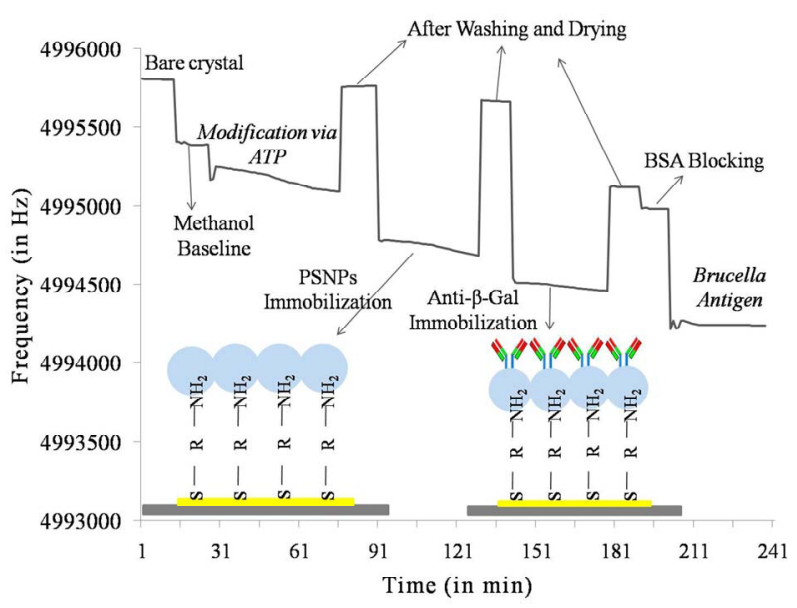

Figure 6. Frequency shift monitored to check the specificity and stability of immobilized antibodies on PS Nanoparticles surface

crystal was washed with methanol to remove the unattached ATP and its frequency, once dried, was found to be $4995757.41 \pm 1.77 \mathrm{~Hz}$. The following step involved immobilization of PSNPs by exposing the sensor surface to a solution of the polystyrene nanoparticles. As before the immobilization was monitored for 1 hour using the QCM system and continued at $4^{\circ} \mathrm{C}$ for $\sim 12 \mathrm{hrs}$. Then the crystal was washed with methanol to remove the unattached PSNPs and the frequency of the dried crystal was again monitored and was found to be $4995665.43 \pm 3.50$ $\mathrm{Hz}$. The subsequent immobilization of Anti $\beta$-Gal antibodies diluted in PBS on PSNPs produced a further decrement of the sensors frequency. Also in this case the antibody attachment was recorded by monitoring the frequency for $1 \mathrm{hr}$ (Figure 6) and then the immobilization was continued for further $15 \mathrm{hrs}$ at $4^{\circ} \mathrm{C}$. The crystal was washed with methanol and PBS to remove excess antibodies and an average frequency of $4995117.83 \pm$ $2.78 \mathrm{~Hz}$ was obtained. Blocking of the free sites of the sensor surface by $1 \%$ BSA for an hour resulted in further decrease in frequency of the dried crystal (4994982.03 \pm $3.09 \mathrm{~Hz}$ ).

To check the specificity and selectivity of the biosensors, the quartz crystal modified with PSNPs and anti $\beta$-Gal antibody was exposed to a PBS solution containing Brucella antigens $(5 \mu \mathrm{g} / \mathrm{ml})$. The frequency of the sensor, recorded in real time by QCM did not show any significant shift. The change in frequency associated with this step was of small magnitude and almost negligible (Figure 6). This is a clear indication that the biosensor developed is not affected by unspecific binding and that the indeed the antibody immobilized on PSNPs are still specific for the correspondent antigen. All the frequency shifts and the corresponding standard deviation for experiment repeated three times are reported in Table 1. 


\section{Conclusion}

The importance of polymeric nanoparticles is increasing as they are replacing metallic nanoparticles in various nanotechnological applications in the pharmaceutical industries, sensor technology, sputtering etc due to their low cost and easiness of preparation. In this work, microemulsion polymerization was used for the synthesis of PSNPs and their performance in sensors in terms of behavior was compared with that of gold nanoparticles (AuNPs), also synthesized in house. The size of PSNPs and AuNPs was determined using TEM and production was confirmed by FTIR. Both types of nanoparticles were then used in a piezoelectric transducer, which offers the possibility to develop specific and sensitive non labeled immunosensors. The comparison of the two types of nanoparticles has shown that both can enhance the surface area of the sensor. In addition, whereas the antibody was firmly attached to the AuNPs, the loose immobilization on the PSNPs resulted in a displacement of the antibody when exposed to a solution of the correspondent antigen. The displacement of the antibody, however, resulted specific, as no effect on the QCM signal was recorded when the PSNPs biosensors was exposed to a solution of the non specific Brucella antigen. Between the two types of nanoparticles we feel that PSNPs are easier to synthesize, more cost effective and eco-friendly. Therefore in the future we will be concentrating on PSNPs based biosensors. Although the displacement signal achieved with the PSNPs could be used for the antigen quantification, our future studies will concentrate in achieving a stronger binding of the antibody, avoiding the problem of detachment during the process of detection. A full characterization in terms of sensitivity and binding kinetics of the PSNPs will also be performed.

\section{Acknowledgements}

Authors acknowledge the receipt of grant provided by Uttarakhand State Council for Science and Technology (UCOST), Dehradun, Uttarakhand (India) for the research work.

\section{REFERENCES}

[1] A. V. Fowler and I. Zabin, "The Amino Acid Sequence of Beta Galactosidase. I. Isolation and Composition of Tryptic Peptides," Journal of Biological Chemistry, Vol. 245, No. 19, 1970, pp. 5032-5041.

[2] B. W. Matthews, "The Structure of $E$. coli $\beta$-Galactosidase," C. R. Biologies. Vol. 328, No. 6, 2005, pp. 549- 556.

[3] L. V. Lukacheva, A. A. Zakemovskaya, E. E. Karyakina, I. N. Zorov, A. P. Sinitsyn, M. V. Sukhacheva, et al., "Determination of Glucose and Lactose in Food Products with the Use of Biosensors Based on Berlin Blue," Jour- nal of Analytical Chemistry, Vol. 62, No. 4, 2007, pp. 388-393.

[4] M. Porsch-Özcürümez, N. Kischel, H. Priebe, W. Splettstösser, E. Finke and R. Grunow, "Comparison of Enzyme-Linked Immunosorbent Assay, Western Blotting, Microagglutination, Indirect Immunofluorescence Assay, and Flow Cytometry for Serological Diagnosis of Tularemia," Clinical and Diagnostic Laboratory Immunology, Vol. 11, No. 6, 2004, pp. 1008-1015.

[5] B. Koenig and M. Graetzel, "A Novel Immunosensor for Herpes Viruses," Analalytical Chemistry, Vol. 66, No. 3, 1994, pp. 341-344.

[6] G. Sauerbrey, "Use of Quartz Crystal Vibrator for Weighting Thin Films on a Microbalance," Zeitschrift für Physik, Vol. 155, No. 2, 1959, pp. 206-222.

[7] K. K. Kanazawa and J. Gordon II, "The Oscillation Frequency of a quartz Resonator in Contact with Liquid," Analytica Chimica Acta, Vol. 175, No. 1, 1985, pp. 99- 105.

[8] D. Kyprianou, A. R. Guerreiro, I. Chianella, E. V. Piletska, S. A. Fowler, K. Karim, et al., "New Reactive Polymer for Protein Immobilisation on Sensor Surfaces," Biosensors and Bioelectronics, Vol. 24, No. 5, 2009, pp. 1365-1371.

[9] S. F. Chen, L. Y. Liu, J. Zhou and S. Y. Jiang, "Controlling Antibody Orientation on Charged Self-Assembled Monolayers," Langmuir, Vol. 19, No. 7, 2003, pp. 2859 2864.

[10] O. Lazcka, F. J. D. Campob and F. X. Mũnoz, "Pathogen Detection: A Perspective of Traditional Methods and Biosensors," Biosensors and Bioelectronics, Vol. 22, No. 7, 2007, pp. 1205-1217.

[11] D. Kyprianou, A. R. Guerreiro, M. Nirschl, I. Chianella, S. Subrahmanyam, A. P. F. Turner, et al., "The Application of Polythiol Molecules for Protein Immobilisation on Sensor Surfaces," Biosensors and Bioelectronics, Vol. 25, No. 5, 2010, pp. 1049-1055.

[12] I. Mannelli, M. Minunni, S. Tombelli and M. Mascini, "Quartz Crystal Microbalance (QCM) Affinity Biosensor for Genetically Modified Organisms (GMOs) Detection," Biosensors and Bioelectronics, Vol. 18, No. 2, 2003, pp. 129-140.

[13] M. Pohanka and P. Skladal, "Piezoelectric Immunosensor for the Direct and Rapid Detection of Francisella tularensis," Folia Microbiologica, Vol. 52, No. 4, 2007, pp. 325-330.

[14] X. Mao, L. Yang, Su X Li and Y. Li, "A Nanoparticle Amplification Based Quartz Crystal Microbalance DNA Sensor for Detection of Escherichia Coli O157:H7," Biosensors and Bioelectronics, Vol. 21, No. 7, 2006, pp. 1178-1185.

[15] M. K. Choudhary, R. Agrawal, R. Kumar, P. Singh, B. R. K. Gupta and K. P. Singh, "Detection of cadmium-resistant Rhizobacteria Using Piezoelectric Nanobiosensor," International Journal of Nanoscience, Vol. 9, No. 5, 2010, pp. 461-469.

[16] R. Colorado Jr., R. J. Villazana and T. R. Lee, "Self Assembled Monolayers on Gold Generated from Aliphatic Dithiocarboxylic Acids," Langmuir, Vol. 14, No. 22, 1998, 
pp. 6337-6340.

[17] A. Tlilli, A. Abdelghani, S. Hleli and M. A. Aaaref, "Electrical Characterization of a Thiol SAM on the Gold as a First Step for the Fabrication of Immunosensors Based on a Quartz Crystal Microbalance," Sensors, Vol. 4, No. 6, 2004, pp. 105-114.

[18] I. Lee, X. Wang, C. F. Zhu, C. Wang and C. Bai, "Investigation of Polystyrene Nanoparticles and DNA-Protein Complexes by AFM with Image Reconstruction," Applied Surface Science, Vol. 126, No. 3-4, 1998, pp. 281-286.

[19] K. K. Kar and P. Parik, "High Molecular Weight Polystyrene Nanospheres," 2005.

[20] E. J. C. Kellar, C. Galiotis and E. H. Andrews, "Raman Vibrational Studies of Syndiotactic Polystyrene. 1. Assignments in a Conformational/Crystallinity Sensitive Spectral Region," Macromolecules, Vol. 29, No. 10, 1996, pp.
3515-3520.

[21] Z. Li, J. Zhou, Z. Zhang and H. Dang, "Self-Assembly of Carboxyl Functionalized Polystyrene Nano-Spheres into Close-Packed Monolayers via Chemical Adsorption," Chinese Journal of Chemistry, Vol. 22, No. 10, 2004, pp. 1133-1137.

[22] V. V. Shmanai, T. A. Nikolayeva, L. G. Vinokurova and A. A. Litoshka, "Oriented Antibody Immobilization to Polystyrene Macrocarriers for Immunoassay Modified with Hydrazide Derivatives of Poly(meth)acrylic Acid," BMC Biotechnology, Vol. 1, No. 1, 2001, p. 4. http://www.biomedcentral.com/1472-6750/1/4

[23] W. Qian, D. Yao, F. Yu, B. Xu, R. Zhou, X. Bao and Z. $\mathrm{Lu}$, "Immobilization of Antibodies on Ultraflat Polystyrene Surfaces," Clinical Chemistry, Vol. 46, No. 9, 2000, pp. 1456-1463. 